\title{
Efficiency evaluation and safety monitoring of tailored rapid potassium supplementation strategy for fatal severe hypokalemia
}

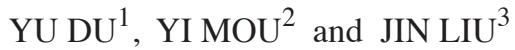 \\ ${ }^{1}$ Emergency Department and Intensive Care Unit, West China School of Public Health, No. 4 West China Teaching Hospital, \\ Sichuan University, Chengdu, Sichuan 610041; ${ }^{2}$ Geroscience and Chronic Disease Department, \\ The 8th Municipal People's Hospital, Chengdu, Sichuan $610083 ;{ }^{3}$ Department of Anesthesia and Intensive Care, \\ West China Hospital, Sichuan University, Chengdu, Sichuan 610041, P.R. China
}

Received August 30, 2017; Accepted January 25, 2019

DOI: $10.3892 /$ etm.2019.7292

\begin{abstract}
Stringent regulations have been established for the intravenous administration of potassium to avoid hyperkalemia in the clinic. The standard approach, however, often does not work well for treating severe hypokalemia. In the present study, a rabbit model of hyperkalemia was used to develop an tailored rapid potassium supplementation strategy and the effectiveness and safety of this new strategy were assessed. A total of 20 rabbits with induced severe hypokalemia were randomly divided into two equal treatment groups. All of the animals were injected with $3 \% \mathrm{KCl}$ through the auricular marginal veins by a micro-injection pump; the target serum potassium concentration was $4 \mathrm{mmol} / \mathrm{l}$. The conventional treatment group was administered a continued potassium infusion at the standard infusion rate of $0.4 \mathrm{mmol} / \mathrm{kg} / \mathrm{h}$. The tailored rapid supplementation group was treated in two steps: First, a loading dose of potassium was rapidly injected for $5 \mathrm{~min}$ and this step was repeated until the serum potassium concentration was increased to $3.5 \mathrm{mmol} / 1$. After this increase in serum potassium concentration, a sustained potassium infusion at a constant dose was performed. Electrocardiogram, blood pressure, respiratory rate, serum potassium concentration, urine volume and vital signs were monitored in real-time. No hyperkalemia occurred in any of the two the groups. However, compared with the conventional group, the tailored rapid group had a significantly shorter duration of potassium infusion and arrhythmia, and a higher survival rate. In conclusion, these results demonstrate that the tailored rapid potassium supplementation strategy shortened the time of hypokalemia and is a safe and better treatment option to remedy life-threatening
\end{abstract}

Correspondence to: $\mathrm{Dr} \mathrm{Yu}$ Du, Emergency Department and Intensive Care Unit, West China School of Public Health, No. 4 West China Teaching Hospital, Sichuan University, 18 Sanduan South Renmin Road, Wuhou, Chengdu, Sichuan 610041, P.R. China E-mail: 456duyu@163.com

Key words: tailored rapid potassium supplementation strategy, fatal severe hypokalemia, efficiency, safety arrhythmia caused by severe hypokalemia with a high success rate.

\section{Introduction}

Severe hypokalemia is a fatal emergent clinical condition in which the serum potassium concentration is $>2.5 \mathrm{mmol} / \mathrm{l}$ (1); it presents as general muscle weakness, cardiac arrhythmia, and respiratory muscle paralysis. Even though this condition is fatal, a swift reversion of the low serum potassium concentration in clinical patients is not allowed due to the strict regulations regarding the supplementation of potassium to prevent pseudohyperkalemia. This approach is followed in most cases; however, it does not work well in cases of severe hypokalemia, particularly when the condition is fatal, e.g., after long-term fasting or insufficient food consumption with severe diarrhea and vomiting (2), poisoning $(3,4)$, after cardiothoracic surgery $(5,6)$, in subjects with hypokalemic periodic paralysis (7) and severe malnutrition. Furthermore, severe hypokalemia often concurs with various critical conditions, including multiple organ failure, severe infection, as well as fluid, electrolyte and acid-base imbalances. These conditions are accompanied with various underlying diseases and complications that require restricted speed and volume of venous infusion. This presents a contradiction: According to the established regulations, to avoid hyperkalemia, the concentration and speed of intravenous potassium supplementation should not exceed $40 \mathrm{mmol} / 1(0.3 \%)$ or $20 \mathrm{mmol} / \mathrm{h}(1.5 \mathrm{~g} / \mathrm{h})$ in adults and $0.4 \mathrm{mmol} / \mathrm{kg} / \mathrm{h}$ in children; furthermore, direct intravenous injection of potassium is strictly prohibited $(1,8)$; however, to correct severe hypokalemia in the presence or absence of critical diseases, rapid infusion of a small volume of potassium solution may be beneficial.

Of note, the American Heart Association (AHA) recommends an empirical intravenous injection of $2 \mathrm{mEq}$ of potassium in the first $1 \mathrm{~min}$, followed by an intravenous infusion of $10 \mathrm{mEq}$ of potassium over 5-10 min in cases where hypokalemia led to malignant ventricular arrhythmia or inevitable cardiac arrest (9). Although several case reports and randomized clinical trial investigations have suggested the success of this novel recommendation $(2,10,11)$, more 
persuasive, evidence-based proof is required. Slow potassium supplementation may not lead to treatment failure, increase the severity of the disease and increase the risk of patient death; however, empirical rapid potassium supplementation is potentially dangerous. In the present study, an tailored rapid potassium supplementation strategy was developed, which was based on the hemodynamic principle, as well as the mechanism of potassium distribution and balance, for calculating the proper concentration, infusion rate and dose of potassium supplement for individuals with bedside monitoring via electrocardiogram (ECG) and the serum potassium concentration. In the era of precise medicine, with exceptional cases and AHA recommendations $(2,8,10,11)$, this tailored rapid potassium supplementation strategy has the potential to become the optimum treatment option in the future. On the basis of an integrated approach, it was hypothesized that this strategy may restore extremely low serum potassium concentrations in patients admitted to emergency departments and intensive care units.

Barium ( $\mathrm{Ba}$ ) is a silver-white alkaline metal that is widely distributed in nature. $\mathrm{Ba}$ is almost non-toxic, but the toxicity of Ba salt is determined by its solubility; Ba chloride $\left(\mathrm{BaCl}_{2}\right)$ is the most toxic soluble Ba salt (12). Ba may induce a low serum potassium concentration via the following two synergistic mechanisms: i) $\mathrm{Ba}$ ions activate $\mathrm{Na}^{+} / \mathrm{K}^{+}$-ATPase in the cell membrane and block the potassium channels; this causes potassium ions to be redistributed inside and outside of the cell, and extracellular potassium reverses the chemical gradient into the cell; ii) the potassium channel blockade makes it difficult for the cell to excrete potassium, resulting in high intracellular and low extracellular potassium, which causes membrane current inhibition and produces a series of clinical manifestations associated with low potassium, including muscle paralysis, respiratory failure and fatal arrhythmia (13). This is also the major cause of death due to acute $\mathrm{BaCl}_{2}$ poisoning (14-16).

The animal model established in the present study is based on the characteristics of $\mathrm{BaCl}_{2}$ poisoning, which not only rapidly causes severe hypokalemia, but also easily induces arrhythmia. The present study first established an animal model of acute fatal severe hypokalemia with $\mathrm{BaCl}_{2}$ and then performed a potassium supplementation experiment using this model. The purpose of the present study was to compare the conventional potassium supplementation method with a newly developed the tailored rapid potassium supplementation strategy in order to evaluate its efficiency and safety, and to provide an experimental basis for the development of an effective, safe and scientific strategy for the clinical rescue of fatal severe hypokalemia.

\section{Materials and methods}

Experimental animals. The Animal Ethics Committee of Sichuan University (Chengdu, China) approved all of the procedures performed in the present study. A total of 30 adult healthy male and female Japanese white rabbits (weight, $2.5 \mathrm{~kg}$; age, 12-14 weeks) were purchased from the Animal Center of Sichuan Province (Chengdu, China), and kept for 3-5 days in single cages. Animals were housed at a temperature of $22-24^{\circ} \mathrm{C}$ and a relative humidity of $30-40 \%$ with free access to food and water and a $12 \mathrm{~h}$ light/dark cycle prior to the start of the experiment.

Establishment of animal disease model. The conditions of the current study are based on preliminary experimental results from acute toxicity experiments assessing barium chloride in rabbits (17). Barium chloride gavage and continuous potassium supplementation monitoring were performed under anaesthesia. All rabbits were $12 \mathrm{~h}$ prior to the beginning of the experiment and ate immediately following potassium supplementation. After weighing, a 22-gauge catheter was inserted and placed in the central ear artery of each rabbit for blood pressure monitoring and blood sampling by connecting a T-branch tube. The rabbits were anesthetized by an intraperitoneal injection of urethane $(1,000 \mathrm{mg} / 5 \mathrm{ml} / \mathrm{kg})$. After successful anesthesia, the rabbit was fixed on the operation bench and a urinary catheter was inserted into the empty bladder. A needle electrode was then inserted and connected to the BL-420E $\mathrm{E}^{+}$Biological Function Experimental System (Chengdu TME Technology Co., Ltd., Chengdu, China) for real-time recording of ECG, blood pressure, respiratory rate and oxygen saturation. $\mathrm{BaCl}_{2}$ [dose at which $50 \%$ of animal mortalities occurred in 14 days $\left(\mathrm{LD}_{50}\right), 168 \mathrm{mg} / 5 \mathrm{ml} / \mathrm{kg}$ ] was delivered by oral gavage. Every 30 min or whenever arrhythmia occurred, blood samples of $0.5 \mathrm{ml}$ were extracted from the central ear artery of the rabbits to determine the serum potassium concentration. When the serum potassium concentration reached $<2.5 \mathrm{mmol} / \mathrm{l}$ and was accompanied with arrhythmia, the fatal severe hypokalemia model was considered established. The experimental rabbits which did not meet the above criteria regarding the serum potassium levels and ECG were ruled out.

Randomized grouping principle. The model animals were randomized using the 'repeated fair coin-tossing method'. A total of 20 rabbits with severe hypokalemia were randomly divided into two treatment groups $(n=10$ per group): Conventional potassium supplementation group (conventional treatment group) and tailored rapid potassium supplementation group (tailored rapid treatment group).

Potassium supplementation methods. Potassium chloride infusion was performed through the auricular marginal vein by a micro-injection pump, and the target serum potassium concentration was $4 \mathrm{mmol} / \mathrm{l}$.

For the conventional treatment group, the total amount of potassium supplement was calculated by the formula [4-actual serum potassium concentration $(\mathrm{mmol} / \mathrm{l})] \mathrm{x}$ weight $(\mathrm{kg}) \mathrm{x} 0.2$; this was infused into the auricular marginal vein at the concentration of $3 \%$ and at the infusion rate of $0.4 \mathrm{mmol} / \mathrm{kg} / \mathrm{h}(18,19)$. If necessary, the potassium deficiency was recalculated and potassium solution was infused to obtain the present concentration. Potassium supplementation was stopped if the target serum potassium concentration was reached or if the animal died in the experiment.

The tailored rapid treatment group was administered a loading dose and a maintenance dose. The loading dose, calculated by the formula [4-actual serum potassium concentration $(\mathrm{mmol} / \mathrm{l})] \mathrm{x}$ weight $(\mathrm{kg}) \mathrm{x} 0.07$, was infused into the auricular marginal vein at a uniform infusion rate within $5 \mathrm{~min}$; this dose was repeated until the serum potassium concentration increased 
to $3.5 \mathrm{mmol} / \mathrm{l}$. Subsequently, a maintenance dose, calculated by the formula [4-actual serum potassium concentration (mmol/l)] x weight $(\mathrm{kg}) \times 0.2$, was injected with a micro-pump at the infusion rate of $0.4 \mathrm{mmol} / \mathrm{kg} / \mathrm{h}$. All the potassium solutions were prepared and infused at a concentration of $3 \%$. In the case where the serum potassium concentration did not increase to the target concentration, the respective steps were repeated for a maintenance dose. Potassium supplementation was stopped if the target serum potassium concentration was reached or if the animal died in the experiment (Fig. 1).

Observation parameters. In the experiment, the serum potassium concentration was measured prior to and at the end of each potassium supplementation cycle until the serum potassium concentration reached the target level. All urine excreted during the potassium supplementation procedure was collected for the measurement of volume and the potassium concentration. The duration of potassium infusion, the amount of potassium infused, mean arterial pressure (MAP), respiratory rate (RR), ECG and adverse effects were recorded. From then on, serum potassium concentrations were measured once per day in the 14-day observation period. In addition, the respiratory rate of rabbits was observed without anaesthesia and ECGs were monitored via electrodes. The death of rabbits was continuously observed following potassium supplementation and no rescue measures were taken prior to mortality, and the serum potassium concentration was determined again before death.

Calculation method for observation parameters. The serum potassium concentration prior to potassium supplementation (mmol/l) was measured after successfully establishing the model, and was defined as the serum potassium concentration before the beginning of potassium supplementation treatment.

The serum potassium concentration after potassium supplementation (mmol/l) was defined as the serum potassium concentration after stopping the treatment or at death of the animal.

The duration of potassium infusion (h) was the time interval of potassium supplementation treatment.

The actual total amount of potassium supplement (mmol) was the total amount of serum potassium ions in the body from the beginning to the end of the potassium supplementation.

The net amount of potassium supplement (mmol) was the actual total amount of potassium supplement- the amount of potassium excreted through the kidneys.

The total urinary volume $(\mathrm{ml})$ was the total urinary volume from the beginning to the end of the potassium supplementation.

The amount of potassium excreted through the kidneys (mmol) was the urine potassium concentration $\mathrm{x}$ the total urinary volume.

The amount of extracellular fluid (ECF) potassium (mmol) was the serum potassium concentration $\mathrm{x}$ the body weight $(\mathrm{kg})$ $\mathrm{x} 0.2$.

The ECF potassium alteration [post- vs. pre-potassium supplementation (mmol)] was the ECF potassium concentration after potassium supplementation-the ECF potassium concentration prior to potassium supplementation.

The amount of the transcellular potassium shift (mmol) was determined as the net amount of potassium
supplementation-ECF potassium alteration (post- vs. pre-potassium supplementation).

The criteria for arrhythmias associated with hypokalemia were as follows: Ventricular arrhythmias, including various types of ventricular pre-mature beats, ventricular tachycardia and ventricular fibrillation.

The duration of cardiac arrhythmia was the time of beginning and disappearance of each arrhythmia.

Sample testing method. The serum potassium concentration was determined using the following method: Blood samples were collected from the central artery of rabbit ears and placed into the ABL800FLEX automatic biochemical-blood gas analyser (Radiometer, Copenhagen, Denmark). The serum potassium concentration was measured using the ion selective electrode method.

The urine potassium concentration was determined as follows: Urine samples were analyzed using the DxcUnicel 800 Synchron automatic biochemical analyser (Beckmann Coulter, Brea, CA, USA) and the samples were measured via the indirect potential method.

Statistical methods. SPSS.16 software was utilized to perform statistical analysis (SPSS, Inc., Chicago, IL, USA). The results for all measurement variables were first tested for normal distribution. Variables with a normal distribution were expressed by arithmetic means with standard deviations, while variables with an abnormal distribution were expressed by the median. Student's t-tests or analysis of variance F-tests (followed by an LSD or SNK post-hoc test) were used to evaluate differences in the mean values of variables with a normal distribution, and the rank-sum test was used to evaluate differences in variables with an abnormal distribution. Categorical variables were summarized by frequency counts with percentages, while the chi-square test or Fisher's exact test were used to evaluate differences between groups. $\mathrm{P}<0.05$ was considered to indicate a statistically significant difference.

\section{Results}

Establishment of the rabbit model of hypokalemia. Following the administration of $\mathrm{BaCl}_{2}$, only 20 in 30 experimental rabbits reached the experimental requirements. The serum potassium concentration of these 20 experimental rabbits had significantly declined at $0.5 \mathrm{~h}(\mathrm{P}<0.05)$, maintained a stable decrease at $1-1.5 \mathrm{~h}(\mathrm{P}<0.05)$ and exhibited a further gradual decrease until $2 \mathrm{~h}$, when it reached $<2.5 \mathrm{mmol} / 1(\mathrm{P}<0.05)$, and arrhythmia occurred (Fig. 2). At this time-point, the establishment of the fatal severe hypokalemia model in the 20 experimental rabbits was considered to be successful, and the potassium supplementation experiments were started.

No significant differences between the two groups in body weight, serum concentration of $\mathrm{K}^{+}, \mathrm{Na}^{+}$and $\mathrm{Cl}^{-}$, blood $\mathrm{pH}$, arterial partial pressure of oxygen and $\mathrm{PCO}_{2}, \mathrm{MAP}$, heart rate and RR were observed $(\mathrm{P}>0.05$; Table I).

Comparison of serum potassium concentration at preand post-potassium supplementation, as well as duration and dose of potassium supplementation between the two groups. The serum potassium concentration prior to the 


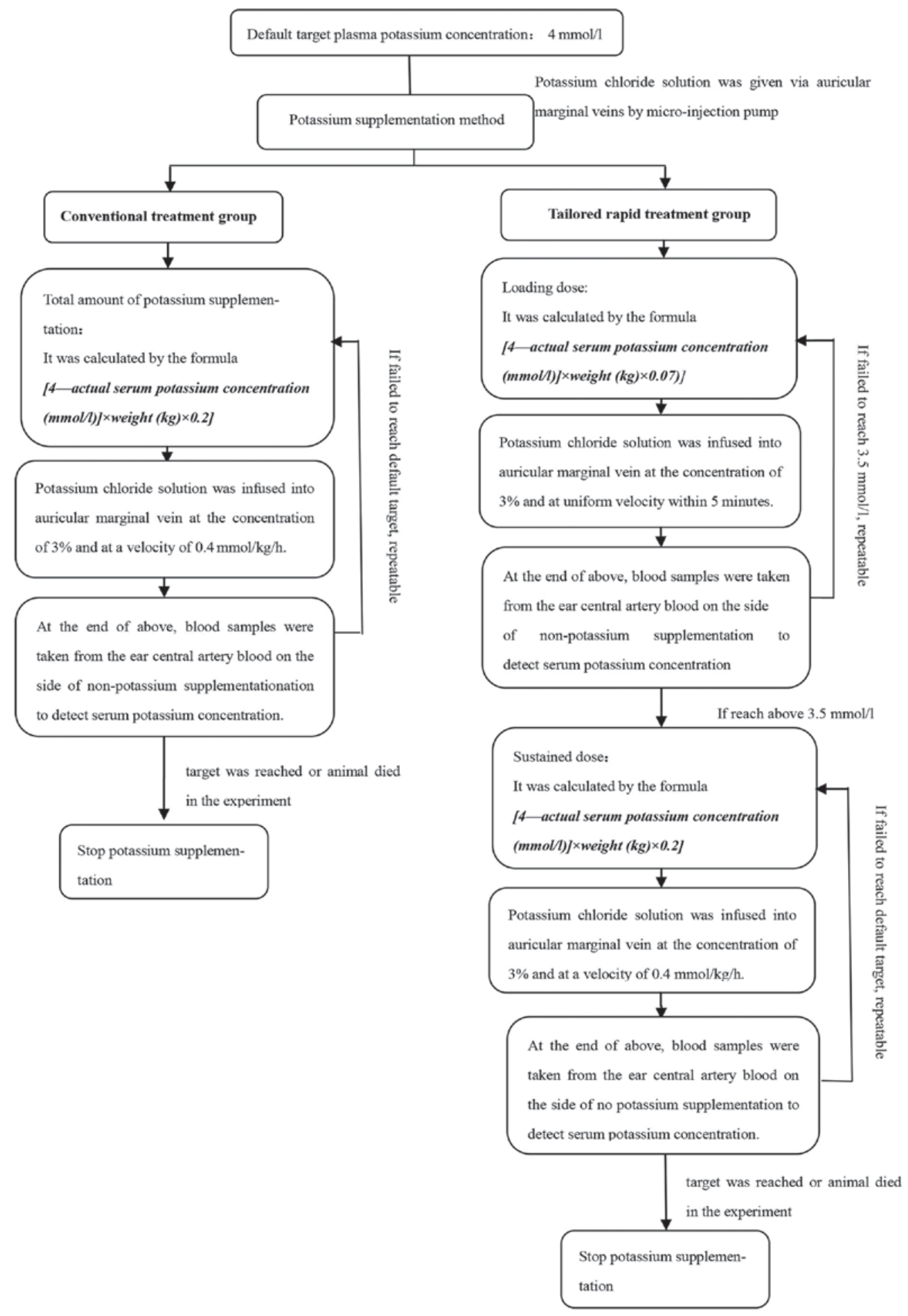

Figure 1. Schematic diagram of potassium supplementation method. A total of 20 rabbits with severe hypokalemia were randomly divided into two treatment groups: Conventional potassium supplementation group (conventional treatment group) and tailored rapid potassium supplementation group (tailored rapid treatment group), with 10 rabbits in each group. These two different methods were used to perform intravenous potassium supplementation in these two groups, and the target potassium concentration was $4 \mathrm{mmol} / \mathrm{l}$.

experiment, the actual amount of potassium supplement and the net amount of potassium supplement were not significantly different between the two groups $(\mathrm{P}>0.05)$. However, the serum potassium concentration after potassium supplementation and the potassium increment post- vs. pre-potassium supplementation in the tailored rapid treatment group were significantly higher than those in the conventional treatment group $(\mathrm{P}<0.05)$; Furthermore, the duration of potassium supplementation in the tailored rapid group was significantly shorter than that in the conventional group $(\mathrm{P}<0.05)$, and the actual amount and the net amount of the potassium supplement per hour in the tailored rapid treatment group were significantly higher than those in the conventional treatment group $(\mathrm{P}<0.05$; Table II). 
Table I. Comparison of the baseline state of the two groups prior to creating the model.

\begin{tabular}{lccr}
\hline Variable & Conventional group $(\mathrm{n}=10)$ & Tailored rapid group $(\mathrm{n}=10)$ & P-value \\
\hline Weight $(\mathrm{kg})$ & $2.4 \pm 0.2$ & $2.4 \pm 0.1$ & 0.990 \\
$\mathrm{Sex}(\mathrm{female} / \mathrm{male})$ & $5 / 5$ & $5 / 5$ & 1.000 \\
$\mathrm{~K}^{+}(\mathrm{mmol} / \mathrm{l})$ & $4.0 \pm 0.4$ & $4.0 \pm 0.7$ & 0.908 \\
$\mathrm{Na}^{+}(\mathrm{mmol} / \mathrm{l})$ & $137.2 \pm 4.5$ & $140.2 \pm 6.1$ & 0.226 \\
$\mathrm{Cl}^{-}(\mathrm{mmol} / \mathrm{l})$ & $108.7 \pm 3.6$ & $106.5 \pm 5.7$ & 0.314 \\
$\mathrm{Blood} \mathrm{pH}$ & $7.4 \pm 0.1$ & $7.4 \pm 0.1$ & 0.977 \\
$\mathrm{PO}_{2}(\mathrm{~mm} \mathrm{Hg})$ & $91.1 \pm 7.9$ & $89.1 \pm 16.4$ & 0.724 \\
$\mathrm{PCO}_{2}(\mathrm{~mm} \mathrm{Hg})$ & $27.2 \pm 4.2$ & $28.9 \pm 0.1$ & 0.338 \\
$\mathrm{MAP}(\mathrm{mm} \mathrm{Hg})$ & $84.2 \pm 6.5$ & $81.8 \pm 7.1$ & 0.376 \\
$\mathrm{Hr}($ beats/min) & $332.1 \pm 24.2$ & $334.7 \pm 17.9$ & 0.185 \\
$\mathrm{RR}($ breaths/min $)$ & $59.4 \pm 9.9$ & $61.1 \pm 7.4$ & 0.399
\end{tabular}

$\mathrm{PO}_{2}$, arterial partial pressure of oxygen; $\mathrm{PCO}_{2}$, arterial partial pressure of carbon dioxide; MAP, mean arterial pressure; Hr, heart rate; RR, respiratory rate.

Comparison of renal potassium excretion during the potassium supplement treatment between the two groups. When the potassium supplementation was completed, because of the short time of potassium supplementation in the tailored rapid treatment group, the urine volume in this group was less than that in the conventional treatment group $(\mathrm{P}<0.05)$, but the average urine volume per hour in the two groups exhibited no significant difference $(\mathrm{P}>0.05)$. Although the urine potassium concentration and renal potassium excretion velocity in the tailored rapid treatment group were higher than those in the conventional treatment group $(\mathrm{P}<0.05)$, the total renal potassium excretion was lower than that in the conventional treatment group $(\mathrm{P}<0.05$; Table II).

Comparison of transcellular potassium shift during the potassium supplement treatment. Prior to supplementation, no significant difference in the ECF potassium concentration was present between the two groups $(\mathrm{P}>0.05)$. After potassium supplementation, the ECF potassium concentration in the tailored rapid treatment group was significantly higher than that in the conventional group $(\mathrm{P}<0.05)$. Although the rate of transcellular potassium shift in the tailored rapid treatment group was higher than that in the conventional treatment group, the total amount of the transcellular potassium shift in the tailored rapid treatment group was lower than that in the conventional treatment group $(\mathrm{P}<0.05$; Table II).

Comparison of the occurrence of arrhythmia after potassium supplementation between the two groups. When cardiac arrhythmia occurred, the serum potassium concentration was not significantly different between the two groups $(\mathrm{P}>0.05)$. However, the duration of cardiac arrhythmia in the conventional treatment group was longer than that in the tailored rapid treatment group $(\mathrm{P}<0.05$; Table II).

Comparison of the changes in circulatory and respiratory parameters pre- and post-potassium supplementation. At the three time-points, namely at baseline, and pre- and post-potassium supplementation, the MAP and RR exhibited

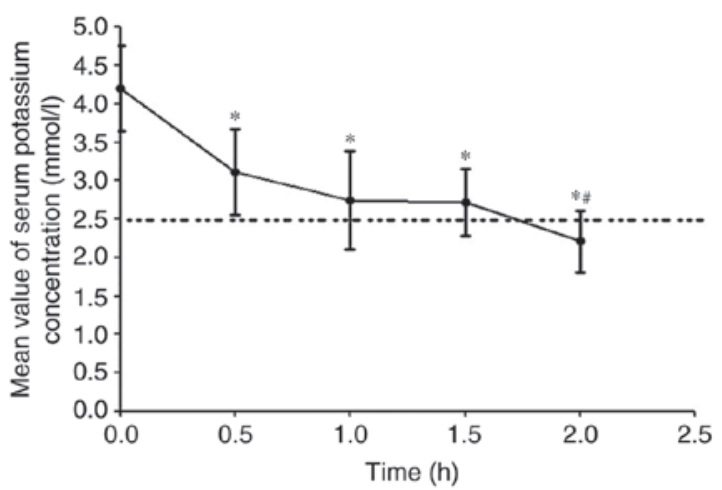

Figure 2. Establishment time to establish the model of fatal severe hypokalemia. The serum potassium concentration is expressed as the mean \pm standard deviation $(\mathrm{n}=20)$. ${ }^{*} \mathrm{P}<0.001$ compared with baseline; ${ }^{*} \mathrm{P}<0.05$ compared with all other time-points.

no significant difference between the two groups $(\mathrm{P}>0.05)$. Furthermore, the MAP prior to the potassium supplementation for each of the two groups was lower than the basal MAP $(\mathrm{P}>0.05)$, and the MAP was elevated after the potassium supplementation $(\mathrm{P}<0.05)$ and became almost equal to the basal MAP ( $>0.05$; Fig. 3). The RR prior to the potassium supplementation was higher than the basal $R R(P<0.05)$, while the RR decreased after potassium supplementation $(\mathrm{P}<0.05)$ and was almost equal to the basal RR $(\mathrm{P}>0.05$; Fig. 4).

Comparison of adverse events and death during the experiment. In each of the two groups, no hyperkalemia occurred during the potassium supplementation process. At the end of the experiment, all of the rabbits $(100 \%)$ in the tailored rapid treatment group were alive, while only six rabbits (60\%) were alive in the conventional treatment group; the cause of death was associated with hypokalemia. Two rabbits died during the course of potassium supplementation, as the increase in the serum potassium concentration was too slow, leading to the occurrence of arrhythmia, respiratory failure and death. 
Table II. Comparison of observation parameters pre- and post-potassium supplementation between the two groups.

\begin{tabular}{|c|c|c|c|}
\hline Variable & $\begin{array}{l}\text { Conventional } \\
\text { group }(n=10)\end{array}$ & $\begin{array}{l}\text { Tailored rapid } \\
\text { group }(\mathrm{n}=10)\end{array}$ & P-value \\
\hline Serum potassium concentration prior to potassium supplementation $(\mathrm{mmol} / \mathrm{l})$ & $2.1 \pm 0.3$ & $2.2 \pm 0.2$ & 0.601 \\
\hline Serum potassium concentration after potassium supplementation $(\mathrm{mmol} / \mathrm{l})$ & $3.6 \pm 0.6$ & $4.6 \pm 0.4$ & 0.001 \\
\hline Potassium increase post- vs. pre-potassium supplementation (mmol/l) & $1.5 \pm 0.8$ & $2.4 \pm 0.3$ & 0.003 \\
\hline Duration of potassium infusion (h) & $4.7 \pm 1.4$ & $2.1 \pm 0.7$ & $<0.001$ \\
\hline Actual total amount of potassium supplement (mmol) & $4.0 \pm 0.9$ & $3.6 \pm 0.4$ & 0.174 \\
\hline Actual infusion rate of potassium supplement (mmol/h) & $0.9 \pm 0.1$ & $1.9 \pm 0.5$ & $<0.001$ \\
\hline Net amount of potassium supplement (mmol) & $2.6 \pm 0.4$ & $2.6 \pm 0.2$ & 0.927 \\
\hline Net infusion rate of potassium supplement $(\mathrm{mmol} / \mathrm{h})$ & $0.6 \pm 0.1$ & $1.4 \pm 0.4$ & $<0.001$ \\
\hline Total urine volume $(\mathrm{ml})^{\mathrm{a}}$ & $13.6 \pm 4.7$ & $6.4 \pm 1.8$ & $<0.001$ \\
\hline Rate of urine output $(\mathrm{ml} / \mathrm{h})$ & $2.9 \pm 0.6$ & $3.2 \pm 0.3$ & 0.177 \\
\hline Urine potassium concentration $(\mathrm{mmol} / \mathrm{l})$ & $108.4 \pm 19.7$ & $164.9 \pm 18.1$ & $<0.001$ \\
\hline Amount of renal potassium excretion (mmol) & $1.5 \pm 0.5$ & $1.0 \pm 0.3$ & 0.037 \\
\hline Rate of renal potassium excretion (mmol/h) & $0.3 \pm 0.1$ & $0.5 \pm 0.1$ & $<0.001$ \\
\hline Amount of ECF potassium before potassium supplementation (mmol) & $1.0 \pm 0.2$ & $1.1 \pm 0.1$ & 0.899 \\
\hline Amount of ECF potassium after potassium supplementation (mmol) & $1.8 \pm 0.3$ & $2.2 \pm 0.2$ & 0.001 \\
\hline $\begin{array}{l}\text { ECF potassium post- vs. pre-potassium supplementation } \\
(\mathrm{mmol})\end{array}$ & $0.7 \pm 0.4$ & $1.2 \pm 0.2$ & 0.002 \\
\hline Amount of transcellular potassium shift (mmol) & $1.8 \pm 0.6$ & $1.4 \pm 0.2$ & 0.043 \\
\hline Rate of the transcellular potassium shift $(\mathrm{mmol} / \mathrm{h})$ & $0.4 \pm 0.2$ & $0.8 \pm 0.3$ & 0.003 \\
\hline Serum potassium concentration at the occurrence of arrhythmia ( $\mathrm{mmol} / \mathrm{l})$ & $2.1 \pm 0.3$ & $2.1 \pm 0.4$ & 0.769 \\
\hline Duration of cardiac arrhythmia (min) & $71.8 \pm 9.8$ & $19.6 \pm 8.9$ & $<0.001$ \\
\hline Animals with occurrence of hyperkalemia (n) & 0 & 0 & 1.000 \\
\hline Surviving animals $(\mathrm{n})$ & 6 & 10 & 0.043 \\
\hline Total number of bleeds in 14-day period (n) & $18.4 \pm 7.2$ & $25.7 \pm 3.7$ & 0.010 \\
\hline Total blood volume withdrawn from the animals in 14-day period (ml) & $9.3 \pm 3.6$ & $13.1 \pm 1.9$ & 0.010 \\
\hline
\end{tabular}

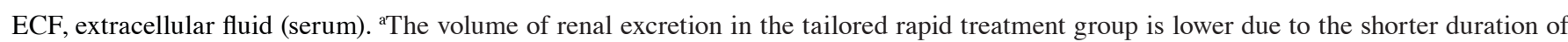
infusion.

Table III. Mortality of rabbits in the conventional treatment group.

\begin{tabular}{ccccc}
\hline Subject no. & $\begin{array}{c}\text { Duration of potassium } \\
\text { infusion }(\mathrm{h})\end{array}$ & Time of death $(\mathrm{h})$ & $\begin{array}{c}\text { Serum potassium concentration at death } \\
(\mathrm{mmol} / \mathrm{l})\end{array}$ & Cause of death \\
\hline 1 & 3.6 & 3.6 & 2.5 & $\mathrm{VT}, \mathrm{VF}, \mathrm{RF}$ \\
4 & 4 & 24.3 & 2.4 & $\mathrm{VF}, \mathrm{RF}$ \\
8 & 3.6 & 3.6 & 2.9 & $\mathrm{VT}, \mathrm{VF}$ \\
10 & 4.3 & 28.5 & 2.2 & $\mathrm{VT}, \mathrm{VF}, \mathrm{RF}$ \\
\hline
\end{tabular}

VT, ventricular tachycardia; VF, ventricular fibrillation; RF, respiratory failure.

Another two rabbits died at 1 day after the end of the potassium supplementation, as the serum potassium concentration decreased, leading to death. In addition, in the 14-day observation period, the total number of bleeds for the rabbits in the conventional treatment group was $18.4 \pm 7.2$ times and the total blood volume withdrawn during multiple blood draws was $0.004 \pm 0.0011 / \mathrm{kg}$, but those in the tailored rapid treatment group were $25.7 \pm 3.7$ times and $0.005 \pm 0.0011 / \mathrm{kg}$, respectively (Tables II and III).

\section{Discussion}

In clinical practice, it is difficult to correct fatal severe hypokalemia via conventional potassium supplementation. In the potassium supplement experiments of the current study, the dose/speed of potassium administered in the conventional treatment group may have been too low, leading to the mortality of 4/10 animals. This serves as a limitation of conventional therapy and should be considered when designing a novel 


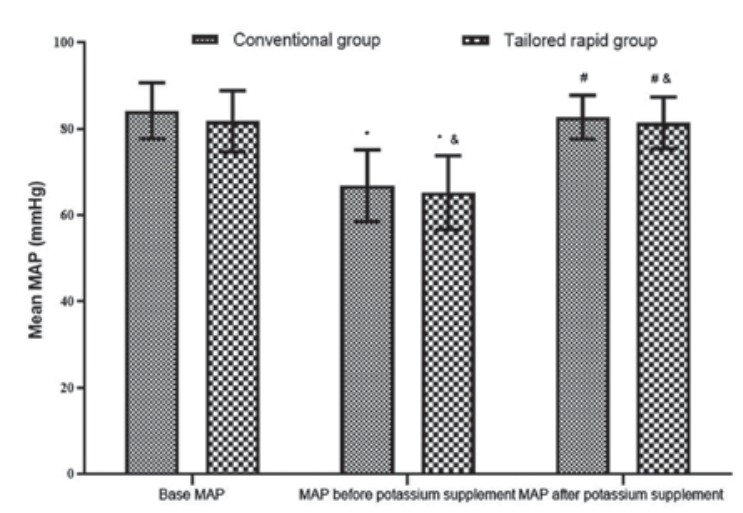

Figure 3. Comparison of MAP pre- and post-potassium supplementation between the two groups. Values are expressed as the mean \pm standard deviation $(\mathrm{n}=20)$. ${ }^{\mathrm{P}}<0.001$ vs. the base MAP of the conventional group; ${ }^{*} \mathrm{P}<0.001$ vs. the MAP prior to potassium supplementation of the conventional group; ${ }^{*} \& \mathrm{P}<0.001$ vs. the base MAP of the tailored rapid group; ${ }^{\#, \&} \mathrm{P}<0.001$ vs. the MAP prior to potassium supplementation of the tailored rapid group.

strategy in future experimentation. The feasibility to pursue the strategy of 'tailored rapid potassium supplementation' to achieve rapid reversal of the fatal condition of severe hypokalemia was explored in the present study. For this, a novel rabbit model of $\mathrm{BaCl}_{2}$-induced severe hypokalemia was established, which was subjected to conventional treatment or a novel and more rapid treatment strategy comprised of initial bolus injections, followed by maintenance infusion.

First, the required dose of potassium supplement that rapidly increases the serum potassium concentration to the required level through the circulation was calculated. The occurrence of cardiac arrest is determined by the extracellular potassium concentration of cardiomyocytes, which is more likely to be influenced by the speed and location of potassium infusion $(2,8,10,11)$, and it also determines the tolerance of the heart to rapid potassium supplementation (11). The body's circulation volume is $\sim 7 \%$ of the body weight (20). For an adult with a body weight of 60-100 kg, the circulation volume would be 4-7 1. The stroke volume of a normal adult in the resting state is $65-70 \mathrm{ml}$; thus, for a heart rate of $60-100$ beats $/ \mathrm{min}$, the cardiac minute volume is 4-7 1. However, the circulatory blood volume of rabbits is $\sim 7-10 \%$ of body weight (21) and the cardiac output of rabbits weighing $2.6 \mathrm{~kg}$ is $\sim 0.28 \mathrm{l} / \mathrm{min}$ (22), which is close to the proportions of humans. In general, the cardiac output is approximately equal to the circulation volume; thus, theoretically, injection of potassium supplement at a dose of [(target serum potassium concentration - actual serum potassium concentration) $\mathrm{x}$ circulation volume] within 1 min does not cause hyperkalemia. Therefore, to ensure uniform distribution of potassium in the blood, the experimental design of the present study comprised an intravenous bolus of potassium supplement over $5 \mathrm{~min}$ to ensure safety to the heart.

According to the principles of hemodynamics, circulating blood has an obvious dilution effect on a high potassium concentration. Before the potassium chloride solution infused from the peripheral vein or the central vein reaches the left heart, it follows the blood circulation path involving the vena cava, right atrium and right ventricle, pulmonary artery, pulmonary vein and left heart, as well as the aorta and its branches. Due to the presence of a capillary network in the entire body, together with the elasticity of blood vessels, muscle compression, pump

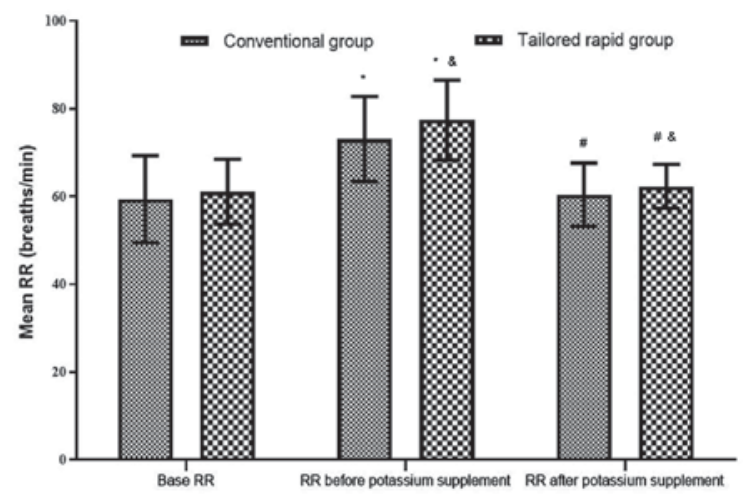

Figure 4. Comparison of RR pre- and post-potassium supplementation between the two groups $(n=20)$. Values are expressed as the mean \pm standard deviation $(n=20) .{ }^{*} \mathrm{P}<0.05$ vs. the base $\mathrm{RR}$ of the conventional group; ${ }^{\text {}} \mathrm{P}<0.05$ vs. the RR prior to potassium supplementation of the conventional group; ${ }^{*}{ }^{*} \mathrm{P}<0.001$ vs. the base RR of the tailored rapid group; ${ }^{\sharp, \&} \mathrm{P}<0.001 \mathrm{vs}$. the RR prior to potassium supplementation of the tailored rapid group.

suction and pressure changes due to the respiratory movement of the thorax, the potassium chloride solution in the blood flows via blood vessels, and it is repeatedly diluted and mixed with the blood. Therefore, even if the potassium chloride solution is directly injected, following which it flows from small blood vessels, a time lag occurs before it reaches the heart; by the time the solution reaches the heart, the potassium chloride concentration of the initial injection is reduced. In addition, in the circulating blood, a certain amount of the potassium ions is taken up by red blood cells and alveolar cells to compensate for potassium deficits; furthermore, certain potassium ions are taken up by other tissue cells for energy metabolism, and a part of the potassium ions is discharged in situ. This continuous process and the process of dynamic reduction further gradually reduces the concentration of potassium from the initial injection. Thus, while the concentration of potassium at the time of injection may be relatively high, it becomes diluted by the time it reaches the heart and does not cause any hyperkalemia. For instance, in the present study, the lowest cardiac output per minute of a normal adult in the resting state (4 l) was assumed to make a calculation for continuous intravenous infusion of potassium solution at the infusion rate of $20 \mathrm{mmol} / \mathrm{h}$, in theory, the amount of potassium that reaches the heart is increased by only $0.083 \mathrm{mmol} / \mathrm{l}$. The same cardiac output, which was injected with $20 \mathrm{mmol}$ potassium for $5 \mathrm{~min}$, reached the heart at only $0.42 \mathrm{mmol} / \mathrm{l}$. The dilution effect of circulating blood to reduce the high potassium concentration is thereby sufficiently explained. With this regard, for calculating the appropriate load to treat severe hypokalemia, the following formula was used: (Target potassium concentration - measured potassium concentration) $\mathrm{x} 0.07$. An intravenous injection of this dose was given over $5 \mathrm{~min}$, which is a feasible approach. The lowest limit of the normal serum potassium concentration is $3.5 \mathrm{mmol} / 1$, which is a relatively safe concentration $(1,23)$. Therefore, it may be reasonable to increase the serum potassium concentration to $3.5 \mathrm{mmol} / \mathrm{l}$ as soon as possible, in order to abrogate all types of fatal complications, including arrhythmia, and reduce the risk of mortality for the patient in a timely manner. Although the tailored rapid potassium supplementation strategy of the current study violates the traditional speed and dosage of 
potassium administration, it is feasible for use in fatal severe hypokalemia. Furthermore, as the circulatory blood volume and cardiac output of rabbits are similar to that of humans the current study may be converted for human use.

After the serum potassium concentration of $3.5 \mathrm{mmol} / \mathrm{l}$ was reached, a maintenance dose of potassium supplement was given for continued correction of the extracellular potassium deficiency and to achieve a balance of potassium in the body fluids. The maintenance dose was calculated using the following formula: (Target serum potassium concentration - actual serum potassium concentration) $\mathrm{x}$ weight $(\mathrm{kg}) \mathrm{x} 0.2$. The normal amount of fluid in the human body accounts for $\sim 60 \%$ of the body weight, and the body fluid is distributed as intracellular and extracellular fluids, which account for 40 and 20\%, respectively. Most of the intracellular fluids are present in the skeletal muscle, while the extracellular fluids comprise plasma and interstitial fluid (5 and $15 \%$ of the body weight, respectively). Potassium ions are mainly distributed in three relatively independent regions: Intracellular fluid pool, blood circulation pool and tissue space pool. As the osmotic equilibrium of potassium between the blood circulation pool and the tissue space is adjusted rapidly, occurring within a few minutes, these areas are considered as one. In clinical practice, the ratio of the potassium concentration pre-potassium injection vs. baseline was calculated from the serum potassium concentration and the body weight, assuming that the ECF accounts for $20 \%$ of the body weight. The amount of potassium deficiency in the ECF was thereby determined and subsequently corrected.

Real-time monitoring and real-time decision making were performed during the present study. With the micro-injection pump and ECG monitor, and the extensive use of point-of-case testing, the infusion rate and concentration of the infused potassium solution was adjusted according to real-time serum potassium concentration to pursue the 'tailored rapid potassium supplementation' strategy. In clinical practice, the amount of potassium supplement is calculated by the potassium deficiency in the ECF, and due to the urinary excretion of a proportion of the potassium dose, it is actually still difficult to reach the set target $(1,24,25)$. In the present study, it was not possible to measure or estimate the amount of intracellular and transcellular potassium shift directly, and in previous clinical studies, the effect on potassium excretion from the kidneys and the clinical manifestations and severity of hypokalemia were different, and the excretion and distribution of potassium may have been influenced by drugs. Therefore, after the calculated supplementary dose is given, the serum potassium concentration should be detected in the peripheral blood vessels in a different location from that of the potassium injection, and the dose should be accordingly adjusted until it reaches the target.

The three major factors that affect potassium supplementation are potassium intake, potassium excretion and transcellular potassium shift. Intravenous potassium supplementation is still the first choice to rescue patients with fatal hypokalemia. Usually, critical patients who cannot tolerate oral potassium and have poor cardiopulmonary function are encountered in the clinic, which restricts the quantity and speed of infusion. Therefore, these conventional methods are not able to elevate the serum potassium concentration to a safe level in a short time, but rather increase the heart capacity load. More importantly, due to the slow infusion according to current restrictions, it takes a long time to treat patients with fatal hypokalemia, and the potassium concentration may not be sufficiently increased within the time-window during which rescue is possible. In the present experimental animal study, the duration of potassium supplementation in the tailored rapid treatment group $(2.1 \pm 0.7 \mathrm{~h})$ was significantly shorter than that in the conventional treatment group $(4.7 \pm 1.4 \mathrm{~h})$. The actual infusion rate $(1.9 \pm 0.5 \mathrm{mmol} / \mathrm{h})$ and the net infusion rate $(1.4 \pm 0.4 \mathrm{mmol} / \mathrm{h})$ of the potassium supplementation in the tailored rapid group were significantly higher than those in the conventional group $(0.9 \pm 0.1$ and $0.6 \pm 0.1 \mathrm{mmol} / \mathrm{h}$, respectively). This implies that the tailored rapid potassium supplementation is superior to the conventional method in restoring the serum potassium concentration.

The kidney is the major organ for potassium excretion and balance. The body's potassium intake and excretion are always balanced under normal conditions. The amount of potassium excreted by the kidneys fluctuates daily, with variations of $>10$-fold (23); this is mainly determined by the amount of intake. When the intake is reduced, the urinary excretion of potassium decreases, and vice versa; however, even under the condition of severe potassium deficiency, the kidney still pursues the activity of potassium excretion (26-28). In the present study, the renal function in the two groups of rabbits was normal. The urine potassium concentration $(164.9 \pm 18.1 \mathrm{mmol} / \mathrm{l})$ and the potassium excretion rate $(0.5 \pm 0.1 \mathrm{mmol} / \mathrm{h})$ in the tailored rapid group were higher than those in the conventional group $(108.4 \pm 19.7 \mathrm{mmol} / \mathrm{l}, 0.3 \pm 0.1 \mathrm{mmol} / \mathrm{h} ; \mathrm{P}<0.05)$, which was associated with the higher dose of potassium supplement in the former group. This result is similar to those of Bundgaard and Kjeldsen (26) and Lin et al (27). However, in the present study, the total urine volume $(6.4 \pm 1.8 \mathrm{ml})$ and the total renal potassium excretion $(1.0 \pm 0.3 \mathrm{mmol})$ in the tailored rapid treatment group were significantly lower than those in the conventional treatment group $(13.6 \pm 4.7 \mathrm{ml}, 1.5 \pm 0.5 \mathrm{mmol}$; $\mathrm{P}<0.05)$. Due to the shortened time of potassium supplementation in the tailored rapid group, the urine volume and the total amount of potassium excreted from the kidney were reduced; thus, the net amount of potassium in 5 min after rapid potassium supplementation was greatly improved, and therefore, this novel strategy improves the serum potassium concentration more effectively.

The transcellular potassium shift is another important factor that affects potassium supplementation. As numerous factors are involved in the shift, the potassium concentration in the ECF and the amount of potassium excretion from the kidney cannot completely reflect the total potassium loss; therefore, it is scientifically inappropriate to calculate the required total amount of potassium only on the basis of the lack of potassium in the ECF. The present study also indicated that even after potassium supplementation according to the above, the serum potassium concentration did not reach the pre-set target level, which is not only associated with the renal excretion of potassium, but also with the transcellular potassium shift. When the body lacks potassium, the transcellular potassium shift rate increases, and the speed and amplitude of serum potassium concentration increase are reduced; consequently, the potassium injection volume increases significantly, and the rate of potassium infusion is probably underestimated (26). In the present study, the total amount of transcellular potassium shift $(1.4 \pm 0.2 \mathrm{mmol})$ in the tailored rapid treatment group was significantly lower than that in the conventional treatment group $(1.8 \pm 0.6 \mathrm{mmol})$, which 
was due to the shortened time of potassium supplementation. The extracellular potassium concentration and the efficiency of potassium supplementation at the end of the experiment were also increased in the tailored rapid treatment group.

In the present study, complications and the risk of mortality were compared between rabbits with severe hypokalemia subjected to the two treatment strategies. It was observed that the tailored rapid treatment group (19.6 $\pm 8.9 \mathrm{~min})$ had a significantly shorter duration of arrhythmia induced by hypokalemia than the conventional group $(71.8 \pm 9.8 \mathrm{~min})$ and an improved prognosis. At the end of the experiment, the survival rate in the tailored rapid treatment group was $100 \%$, while that in the conventional treatment group was only $60 \%(\mathrm{P}<0.05)$. Certain retrospective studies suggested that intravenous infusion of potassium chloride at high concentrations (1.5-9\%) with high speed $(20 \mathrm{mmol} / \mathrm{h})$ to correct mild to moderate hypokalemia is relatively safe and efficient, and no cases died of low potassium or hyperkalemia in the process of potassium supplementation $(10,17,28,29)$. Hamill et al (10) reported 48 cases of critical adult patients with potassium levels of $<3.5 \mathrm{mmol} / \mathrm{l}$ who received potassium chloride infusions $(20,30$ or $40 \mathrm{mmol}$ in $100 \mathrm{ml}$ normal saline over $1 \mathrm{~h}$ ) for patients with serum potassium levels of $<3.5$ but $>3.2 \mathrm{mmol} / 1$, 3.0-3.2 $\mathrm{mmol} / 1$, and $<3.0 \mathrm{mmol} / \mathrm{l}$, respectively. All hypokalemia patients with normal renal function were effectively treated to increase the serum potassium levels in a safe and dose-dependent manner. In a study by He et al (18), 128 critical patients with hypokalemia (serum potassium $<3.5 \mathrm{mmol} / \mathrm{l}$ ) were randomly divided into the therapy group and the control group, which were given 9 and $1.5 \%$ of potassium chloride solution, respectively, intravenously with the aid of a micropump, with an hourly equal quantity of $\mathrm{KCl}$ in the two groups. All patients tolerated the infusion without evidence of hemodynamic changes, hyperkalemia or acute heart dysfunction, and hypokalemia was efficiently treated. Kruse et al (29) observed the effects of potassium chloride infusion in 495 subjects at a medical intensive care unit with a mean pre-infusion potassium level of $3.2 \mathrm{mmol} / \mathrm{l}$. The infusion sets consisted of $1-8$ consecutive individual infusions, each containing $1.5 \%$ potassium chloride solution at the infusion rate of $20 \mathrm{mmol} / \mathrm{h}$. The mean post-infusion potassium level in all patients was $3.9 \mathrm{mmol} / \mathrm{l}$. No temporally associated life-threatening arrhythmias were noted; however, there were 10 instances of mild hyperkalemia. Kruse et al (30) examined the difference between the infusions through the central and the peripheral vein at the same speed with an average concentration of potassium chloride of $2.9 \mathrm{mmol} / \mathrm{l}$ in 40 patients with hypokalemia. In all subjects, the potassium concentration significantly increased in comparison with the baseline. The mean peak potassium concentration was $3.5 \mathrm{mmol} / 1$. No complications, including arrhythmias or changes in cardiac conduction intervals, occurred (30). In order to observe whether this conventional treatment method is equally effective in the treatment of fatal severe hypokalemia, the present study was performed, and the dose and speed of injection used in the previous patient study were converted according to the animal weight. The present study used the same concentration range $(3 \% \mathrm{KCl})$ and speed of intravenous infusion of potassium chloride $(0.4 \mathrm{mmol} / \mathrm{kg} / \mathrm{h})$ in the conventional group as that recommended by previous studies $(1.5-9 \%$ at $20 \mathrm{mmol} / \mathrm{h})$; however, the results are different from those of the previous clinical study. In the conventional group, the death of two rabbits occurred in the process of potassium supplementation, as the increase of serum potassium was too slow. In the present study, a lower level of serum potassium $(<2.5 \mathrm{mmol} / \mathrm{l})$ was used to define severe hypokalemia; however, the previous conventional method dealing with fatal severe hypokalemia remains insufficient. Similar results have been reported in certain case reports. In a study on 13 children with severe hypokalemia (serum potassium, 0.7-1.5 mmol/1), Welfare et al (2) indicated that hypokalemia was not adjusted and 7 cases died of low serum potassium levels even though the dose and speed of potassium supplement were enhanced by $30 \%$ of the conventional recommendation. Furthermore, the previous studies reported no deaths during potassium supplementation, and did not observe whether hypokalemia or hyperkalemia occurred after potassium supplementation $(10,18,29,30)$. However, the present study observed not only the death of animals during potassium supplementation in the conventional treatment group, but also at 1 day after the end of treatment, which was due to a low serum concentration of potassium. Despite the experimental design of the present study, animal death in the conventional treatment group occurred in the 14-day observation period, indicating that there was still a rebound of hypokalemia after conventional potassium supplementation. As the potassium balance in the intracellular and extracellular fluid after intravenous potassium supplementation requires to be maintained for $15 \mathrm{~h}$ or even longer (1), the kidney continues to excrete potassium. If potassium intake is insufficient, hypokalemia symptoms reoccur. The 2000 Guidelines for Cardiopulmonary Resuscitation and Emergency Cardiovascular Care recommended that if cardiac arrest from hypokalemia is imminent (i.e., malignant ventricular arrhythmias), rapid empirical injection of potassium is required (9), which is supported by several successful clinical case reports $(2,31)$. Welfare et al (2) reported that one case of an 8 month-old patient with the severe hypokalemia $(0.7 \mathrm{mmol} / \mathrm{l})$ survived after he was given a 5 times faster than conventional $(2 \mathrm{mmol} / \mathrm{kg} / \mathrm{h})$ intravenous potassium supplement and an extremely high dosage of potassium $(14.5 \mathrm{mmol} / \mathrm{kg} / \mathrm{day})$, without any sign of hyperkalemia during potassium supplementation (2). In cases of hypokalemia caused by aldosterone-producing adenoma, which were treated with potassium infusion at a speed of $36 \mathrm{mmol} / \mathrm{h}$ for $2 \mathrm{~h}$ and for 5 consecutive days, hyperkalemia did not occur (31). The present experimental design is inspired by the inadequacy of the conventional treatment method and guidelines to improve the efficiency of rescuing fatal severe hypokalemia. The potassium supplementation strategy was designed such that it would increase the concentration of serum potassium rapidly to a safe level $(3.5 \mathrm{mmol} / \mathrm{l})$ by intravenous bolus load of potassium solution, and reverse the fatal condition rapidly, and after that, to decrease the speed of continued supplementation to make up for the loss of extracellular potassium, so that the balance of potassium in the intracellular fluids and ECF is restored. The experimental results of the tailored rapid treatment group of the present study are consistent with these reports $(2,31)$ and the previous hypothesis. In addition, in the present study, blood was repeatedly collected from the central ear artery of rabbits, and the volume of blood taken each time, the total number of bleeds and the total blood volume withdrawn during 
several blood draws over a 14-day period in the two groups were within the safety margins for rabbits, no shock occurred and no fluid replacement was required, and the design of the present study was suitable.

The safety of intravenous rapid infusion of potassium must be ensured, as it may be linked with a risk of hyperkalemia and associated fatal arrhythmia. In the present experimental study, no hyperkalemia occurred in any of the two groups subjected to different potassium supplementation strategies. However, the conventional potassium supplementation strategy was not safe for fatal hypokalemia. The animal experiments of the current study revealed that the mortality rate of the conventional group was $40 \%$, the cause of which is associated with low serum potassium levels and insufficient potassium infusion. Certain studies suggest that aberrations in the serum potassium concentration cause fatal ventricular arrhythmia, which is mainly determined by the potassium levels at the heart, and also depends on the heart's tolerance to potassium, which is closely associated with the speed of potassium supplementation and the location of the infusion, but there is no significant association with the concentration of the potassium infusion $(2,10,11)$. In the present study, potassium solution was injected into rabbits through the ear vein, which is located far from the heart; the step-wise dilution by the circulating blood reduced the potassium concentration that reached the heart. When potassium is rapidly infused, the kidney also rapidly drains it simultaneously; this phenomenon is termed 'more intake, more discharge'. The potassium trans-cellular shift, the non-renal mechanism of the potassium balance, was also another important factor. To maintain the balance of potassium in intracellular fluids and ECF, the speed of transcellular potassium shift may increase rapidly after rapid potassium supplementation. During the process of the dynamic reduction of potassium in the circulation, the concentration of the potassium solution was gradually reduced; thus, no hyperkalemia occurred. The present results indicated that the rapid injection of a high concentration of potassium solution may prevent hypokalemia-associated mortality over a short time, while the low-dose, slow infusion strategy is currently implemented to avoid hyperkalemia, as $40 \%$ of the animals in the conventional group died due to the slow speed of potassium supplementation. It may be indicated that the conventional potassium supplementation strategy overestimates the risk of injecting potassium rapidly, particularly for the treatment of fatal severe hypokalemia.

The treatment mode of potassium supplementation cannot be generalized; specific patients require treatment decision-making based on specific circumstances. It is required to determine an appropriate balance between a low and a high potassium concentration, closely monitor ECG and serum potassium concentration, calculate the dose of potassium supplement and reasonably adjust the treatment; this may save affected patients from life-threatening conditions in a timely manner and improve the success rate of the treatment. The tailored rapid potassium supplementation strategy was also confirmed to be effective and safe in the aforementioned experiment.

In conclusion, the present study demonstrated that the tailored rapid potassium supplementation strategy shortens the time to treat hypokalemia and is a safe and better treatment option to reverse life-threatening arrhythmia caused by severe hypokalemia, with a high rescue success rate. Thus, the present study provided a novel concept on intravenous potassium supplementation, termed 'the tailored rapid potassium supplementation strategy', which provides a more efficient, safer and more reasonable method for clinicians to treat patients with fatal severe hypokalemia.

\section{Acknowledgements}

Not applicable.

\section{Funding}

This study was supported by the fund for applied and popular scientific research projects of the Health and Family Planning Commission of Sichuan, China (grant no. 16PJ255).

\section{Availability of data and materials}

The analyzed data sets generated during the study are available from the corresponding author on reasonable request.

\section{Authors' contributions}

YD and JL conceived the current study. YD and YM performed the experiments, collected the data, performed statistical analysis and wrote the manuscript. The final version of the manuscript was read and approved by all authors, and each author believes that the manuscript represents their honest work.

\section{Ethical approval and consent to participate}

The Animal Ethics Committee of Sichuan University (Chengdu, China) approved all of the procedures performed in this study.

\section{Patient consent for publication}

Not applicable.

\section{Competing interests}

The authors declare that they have no competing interests.

\section{References}

1. Gennari FJ: Hypokalemia. N Engl J Med 339: 451-458, 1998.

2. Welfare W, Sasi $\mathrm{P}$ and English M: Challenges in managing profound hypokalaemia. BMJ 324: 269-270, 2002.

3. Bradberry SM and Vale JA: Disturbances of potassium homeostasis in poisoning. J Toxicol Clin Toxicol 33: 295-310, 1995.

4. Yu D, Yi M and Jin L: Incorrigible hypokalemia caused by barium chloride ingestion. Am J Med Sci 349: 279-281, 2015

5. Schaber DE, Uden DL, Stone FM, Singh A, Katkov H and Bessinger FB: Intravenous $\mathrm{KCl}$ supplementation in pediatric cardiac surgical patients. Pediatr Cardiol 6: 25-28, 1985.

6. Prasad A, Ghodsizad A, Pae W, Singbartl K, Boone J, Zeriouh M, Ruhparwar A, Loebe M, Khorrami GS, Koerner MM and Brehm C: Non-cardiac symptoms of moderate to severe hypokalemia in a patient with a syncardia ${ }^{\mathrm{TM}}$ total artificial heart. Heart Surg Forum 19: E12-E13, 2016.

7. Stedwell RE, Allen KM and Binder LS: Hypokalemic paralyses: A review of the etiologies, pathophysiology, presentation, and therapy. Am J Emerg Med 10: 143-148, 1992.

8. Mandal AK: Hypokalemia and hyperkalemia. Med Clin North Am 81: 611-639, 1997 
9. No authors listed: Guidelines 2000 for cardiopulmonary resuscitation and emergency cardiovascular care. Part 8: advanced challenges in resuscitation: Section 1: Life-threatening electrolyte abnormalities. The American heart association in collaboration with the international liaison committee on resuscitation. Circulation 102 (Suppl 8): I217-I222, 2000.

10. Hamill RJ, Robinson LM, Wexler HR and Moote C: Efficacy and safety of potassium infusion therapy in hypokalemic critically ill patients. Crit Care Med 19: 694-699, 1991

11. Choy AM, Lang CC, Chomsky DM, Rayos GH, Wilson JR and Roden DM: Normalization of acquired QT prolongation in humans by intravenous potassium. Circulation 96: 2149-2154, 1997.

12. Choudhury $\mathrm{H}$ and Cary R: Barium and barium compounds. World Health Organisation, Geneva, 2001.

13. Flomenbaum NE: The clinical basis of medical toxicology, section 1, case studies in toxicologic emergencies, pesticides, rodenticides. In: Goldfrank's Toxicologic Emergencies. Goldfrank LR, Flomenbaum NE, Lewin NA, et al (eds). McGraw Hill Medical Publishing Division, pp1379-1392, New York, NY, 2002.

14. Tsai CY, Tseng CC, Liu SF, Lin MC and Fang WF: Acute barium intoxication following accidental inhalationof barium chloride. Intern Med J 41: 293-295, 2011.

15. Jourdan S, Bertoni M, Sergio P, Michele P and Rossi M: Suicidal poisoning with barium chloride. Forensic Sci Int 119: 263-265, 2001.

16. Bowen LN, Subramony SH, Cheng J, Wu SS and Okun MS Elementary, my dear Dr. Allen: The case of barium toxicity and Pa Ping. Neurology 74: 1546-1549, 2010.

17. Rispin A, Farrar D, Margosches E, Gupta K, Stitzel K, Carr G, Greene M, Meyer W and McCall D: Alternative methods for the median lethal dose $(\mathrm{LD}(50))$ test: The up-and-down procedure for acute oral toxicity. ILAR J 43: 233-243, 2002.

18. He Q, Wang JH, Liu YL, Tang PX, Chang ZG, Du LQ and Huang XF: Study on safety and efficacy of concentrated potassium chloride infusions in critically ill patients with hypokalemia. Zhongguo Wei Zhong Bing Ji Jiu Yi Xue 20: 416-418, 2008.

19. Rastergar A and Soleimani M: Hypokalaemia and hyperkalaemia. Postgrad Med J 77: 759-764, 2001.
20. Yuming W: Blood circulation. In: Physiology. Tinghuai W (ed). Vol 4. 9th edition. People's Health Press, Beijing,BJ, pp85$146,2018$.

21. Experimental Animal Anatomy Writing Group of Nankai University. Anatomy of Experimental Animals. China: People's Education Press 120-121, 1979.

22. Guyton AC, Jones CE and Coleman TG: Normal cardiac output and its variations. Chap 1. In: Circulatory Physiology: Cardiac Output and its Regulation. WB Sauders, London, pp3-20, 1973.

23. Halperin ML and Kamel KS: Potassium. Lancet 352: 135-140, 1998.

24. Thompson CB, Choi C, Youn JH and McDonough AA: Temporal responses of oxidative vs. glycolytic skeletal muscles to $\mathrm{K}^{+}$deprivation: $\mathrm{Na}^{+}$pumps and cell cations. Am J Physiol 276: C1411-C1419. 1999.

25. Muto S: Potassium transport in the mammalian collecting duct. Physiol Rev 81: 85-116, 2001.

26. Bundgaard $\mathrm{H}$ and Kjeldsen $\mathrm{K}$ : Potassium depletion increases potassium clearance capacity in skeletal muscles in vivo during acute repletion. Am J Physiol Cell Physiol 283: C1163-C1170, 2002.

27. Lin SH, Cheema-Dhadli S, Gowrishankar M, Marliss EB, Kamel KS and Halperin ML: Control of excretion of potassium: Lessons from studies during prolonged total fasting in human subjects. Am J Physiol 273: F796-F800, 1997.

28. Hoskote SS, Joshi SR and Ghosh AK: Disorders of potassium homeostasis: Pathophysiology and management. J Assoc Physicians India 56: 685-693, 2008.

29. Kruse JA and Carlson RW: Rapid correction of hypokalemia using concentrated intravenous potassium chloride infusions. Arch Intern Med 150: 613, 1990.

30. Kruse JA, Clark VL, Carlson RW and Geheb MA: Concentrated potassium chloride infusion in critically ill patients with hypokalemia. J ClinPharmacol 34: 1077-1082, 1994.

31. Coruzzi P, Gualerzi M, Parati G, Brambilla L, Brambilla V, Di Rienzo $M$ and Novarini A: Potassium supplementation improves the natriuretic response to central volume expansion in primary aldosteronism. Metabolism 52: 1597-1600, 2003. 